\title{
Unification for darkly charged dark matter
}

\author{
Ayuki Kamada $\odot,{ }^{1}$ Masaki Yamada, ${ }^{2}$ and Tsutomu T. Yanagida ${ }^{3,4}$ \\ ${ }^{1}$ Center for Theoretical Physics of the Universe, Institute for Basic Science (IBS), \\ 55 Expo-ro, Yuseong-gu, Daejeon 34126, Korea \\ ${ }^{2}$ Institute of Cosmology, Department of Physics and Astronomy, Tufts University, \\ 574 Boston Avenue, Medford, Massachusetts 02155, USA \\ ${ }^{3}$ T. D. Lee Institute and School of Physics and Astronomy, Shanghai Jiao Tong University, \\ 800 Dongchuan Road, Shanghai 200240, China \\ ${ }^{4}$ Kavli IPMU (WPI), UTIAS, The University of Tokyo, 5-1-5 Kashiwanoha, Kashiwa, \\ Chiba 277-8583, Japan
}

(Received 20 August 2019; accepted 1 July 2020; published 16 July 2020)

\begin{abstract}
We provide a simple UV theory for Dirac dark matter with a massless Abelian gauge boson. We introduce a single fermion transforming as the 16 representation in the $\mathrm{SO}(10)^{\prime}$ gauge group, which is assumed to be spontaneously broken to $\mathrm{SU}(5)^{\prime} \times \mathrm{U}(1)^{\prime}$. The $\mathrm{SU}(5)^{\prime}$ gauge interaction becomes strong at an intermediate scale, and then we obtain a light composite Dirac fermion with $\mathrm{U}(1)^{\prime}$ gauge interaction at a low-energy scale. Its thermal relic can explain the observed amount of dark matter consistently with other cosmological and astrophysical constraints. The dark matter mass and $\mathrm{U}(1)^{\prime}$ fine-structure constant are predicted to be $600-700 \mathrm{GeV}$ and $(2.5-2.9) \times 10^{-2}$, respectively. We discuss that a nonzero kinetic mixing between the $\mathrm{U}(1)^{\prime}$ gauge boson and the hypercharge gauge boson is allowed and the temperature of the visible sector and the dark matter sector can be equal to each other.
\end{abstract}

DOI: 10.1103/PhysRevD.102.015012

\section{INTRODUCTION}

Constructing a grand unified theory (GUT) of the Standard Model (SM) is an outstanding challenge in particle physics. The similarity of the SM gauge coupling constants and the beautiful unification of fermions in the SU(5) multiplets may support the existence of the unified theory at a very high energy scale. However, the running of the gauge coupling constants and the quark-lepton mass relation are deviated from the simplest SU(5) GUT prediction [1-5], which may imply that the GUT breaking in the visible sector is much more complicated than we expect.

In the context of cosmology, there exists dark matter (DM), which may be a fundamental particle that barely interacts with the SM particles. Since the DM must be stable and neutral under the electromagnetic interaction, we consider it to be charged under a dark $\mathrm{U}(1)^{\prime}$ gauge symmetry. Then one may hope that the dark sector is also unified into a GUT' theory as in the SM sector.

In this paper, we propose a chiral $\mathrm{SO}(10) \times \mathrm{SO}(10)^{\prime}$ GUT as a unified model of SM and DM sectors. The first

Published by the American Physical Society under the terms of the Creative Commons Attribution 4.0 International license. Further distribution of this work must maintain attribution to the author(s) and the published article's title, journal citation, and DOI. Funded by SCOAP.
$\mathrm{SO}(10)$ gauge theory is a standard $\mathrm{SO}(10)$ GUT model, which we do not specify as it has been extensively discussed in the literature [6-11]. We focus on the second $\mathrm{SO}(10)^{\prime}$ gauge theory, which gives a dark sector. The fermionic matter content in $\mathrm{SO}(10)^{\prime}$ is a single field in the 16 representation. The $\mathrm{SO}(10)^{\prime}$ is assumed to be spontaneously broken to $\mathrm{SU}(5)^{\prime} \times \mathrm{U}(1)^{\prime}$ at a very high energy scale, and the $\mathrm{SU}(5)^{\prime}$ gauge interaction becomes strong at the energy scale of the order of $10^{13} \mathrm{GeV}$. Below the confinement scale, we have a light composite Dirac fermion charged under the remaining $\mathrm{U}(1)^{\prime}$. Therefore, the DM sector results in Dirac DM with a massless $\mathrm{U}(1)^{\prime}$ gauge boson, which has been discussed in Refs. [12,13]. ${ }^{1}$ A similar idea of the strong $\mathrm{SU}(5)^{\prime}$ gauge theory was used in the literature in different contexts [15-17], where they did or did not introduce the $\mathrm{U}(1)^{\prime}$ gauge symmetry.

As discussed in Ref. [13], DM with a massless dark photon is still allowed by any astrophysical observations and DM constraints even if it is the dominant component of DM. The thermal relic abundance of the Dirac fermion can explain the observed amount of DM. We find that the temperatures of SM and DM sectors can be the same as each other at a high temperature. This allows us to consider a nonzero kinetic mixing between the $\mathrm{U}(1)^{\prime}$ and $\mathrm{U}(1)_{Y}$

\footnotetext{
${ }^{1}$ For scalar DM with a massless or massive $\mathrm{U}(1)^{\prime}$ gauge boson, see Ref. [14].
} 
gauge bosons, which presents an interesting possibility for the DM search in this model. The relic of the massless $\mathrm{U}(1)^{\prime}$ gauge boson affects the expansion rate of the Universe as dark radiation, which can be checked by detailed measurements of the cosmic microwave background $(\mathrm{CMB})$ anisotropies in the future.

\section{DARK MATTER IN THE LOW-ENERGY DYNAMICS}

We first explain a low-energy phenomenology in the dark sector. Let us introduce a $\mathrm{U}(1)^{\prime}$ gauge symmetry and a Dirac fermion $\eta$ of weak-scale mass $m_{\eta}$ with charge $q$. We consider the case where the $\mathrm{U}(1)^{\prime}$ gauge symmetry is not spontaneously broken and the gauge boson $\gamma^{\prime}$ is massless until present. We denote the temperature of the dark sector as $T^{\prime}$ and that of the visible sector as $T$. We define $\xi(T)=T^{\prime} / T$, which depends on the temperature. We will see that there is a viable parameter region even if $\xi=1$ at a high temperature.

The DM can annihilate into a dark photon, and, hence, its thermal relic density is determined by the freeze-out process. The thermally averaged annihilation cross section is given by

$$
\left\langle\sigma v_{\mathrm{Mol}}\right\rangle=\frac{\pi q^{4} \alpha^{2}}{m_{\eta}^{2}} \bar{S}_{\mathrm{ann}}\left(\alpha^{\prime}\right),
$$

where $v_{\text {Mol }}$ is Møller velocity and $\bar{S}_{\text {ann }}$ is the thermally averaged Sommerfeld enhancement factor $[18,19]$. In the regime where the gauge interaction is relatively large, a bound-state formation is efficient and is relevant to determine the thermal relic abundance. Hence, we have to solve the coupled Boltzmann equations for the unbound and bound DM particles as done in Ref. [19]. In Fig. 1, we quote their result to plot a contour on which we can explain the observed amount of DM for the case of $\xi(T)=1$ at the time of DM freeze-out.

The DM has a self-interaction mediated by the dark photon. Its cross section is given by

$$
\begin{aligned}
\frac{\sigma_{T}}{m_{\eta}} & =\frac{8 \pi \alpha^{\prime 2}}{m_{\eta}^{3} v^{4}} \log \Lambda \\
& \simeq 0.2 \mathrm{~cm}^{2} / \mathrm{g}\left(\frac{q^{2} \alpha^{\prime}}{0.025}\right)^{2}\left(\frac{m_{\eta}}{1 \mathrm{TeV}}\right)^{-3}\left(\frac{v}{300 \mathrm{~km} / \mathrm{s}}\right)^{-4},
\end{aligned}
$$

where $\log \Lambda(\approx 40-70)$ comes from an infrared cutoff for the scattering process. The velocity of DM $v$ depends on the scale we are interested in: $v \sim 30,300$, and $1000 \mathrm{~km} / \mathrm{s}$ for dwarf galaxies, galaxies, and galactic clusters, respectively. The observed triaxial structure of galaxy NGC720 puts a stringent upper bound on the self-interaction cross section, since the DM velocity distribution is randomized and is more isotropic by the self-interaction $[12,13,20]$. This can

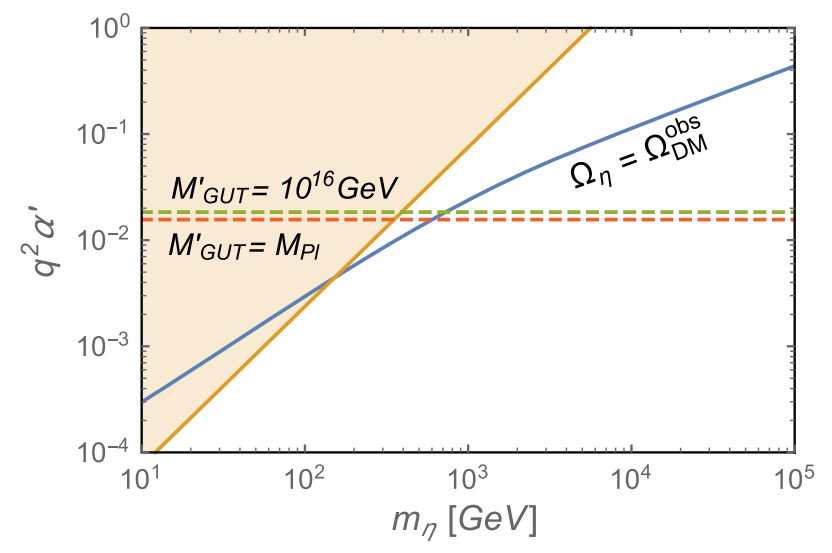

FIG. 1. Constraint on $q^{2} \alpha^{\prime}$ as a function of $m_{\eta}$. We can explain the observed amount of DM on the solid blue curve when $\xi=1$ at the time of DM freeze-out. The orange shaded region is excluded by the ellipticity constraint on the observed galaxy. On the upper and lower dashed lines, the gauge coupling constant can be unified with that of the $\mathrm{SU}(5)^{\prime}$ gauge symmetry at the energy scales of $M_{\mathrm{GUT}}^{\prime}=10^{16} \mathrm{GeV}$ and the Planck scale, respectively, for the case of $q=\sqrt{10} / 4$.

be rewritten as a constraint on the gauge coupling constant and is shown as the orange shaded region in Fig. 1. The DM with mass of the order of $0.1-10 \mathrm{TeV}$ is allowed even if $\xi=1$ at the time of freeze-out, depending on $q^{2} \alpha^{\prime}\left(\gtrsim 10^{-2}\right)$. We expect that a larger number of statistical samples of galactic structures will make the analysis more robust.

Since the self-interacting cross section is proportional to $v^{-4}$, the cross section for the cluster scales is much smaller than the observational constraints [21]. On the other hand, the self-interaction is quite large in smaller scales, like dwarf galaxies. It has been discussed that a too large scattering cross section leads to a very short mean-free path, which suppresses heat conduction and, hence, both core formation and core collapse are inhibited [22,23]. Therefore, the constraint on the dwarf galactic scales may not be applied to this kind of model, and the massless mediator is still allowed for the self-interacting DM model.

The massless dark photon remains in the thermal plasma in the dark sector and contributes to the energy density of the Universe as dark radiation. Its abundance is conveniently described by the deviation of the effective neutrino number from the SM prediction such as

$$
\Delta N_{\mathrm{eff}}=\frac{8}{7}\left(\frac{2}{g_{*}^{\prime}\left(T_{\mathrm{d}}^{\prime}\right)} \frac{g_{*}\left(T_{\mathrm{d}}^{\prime}\right)}{43 / 4}\right)^{-4 / 3} \xi^{4}\left(T_{\mathrm{d}}^{\prime}\right),
$$

where $g_{*}^{\prime}$ is the effective number of degrees of freedom in the dark sector and $T_{\mathrm{d}}^{\prime}$ is the decoupling temperature of the dark sector from the SM sector. In the case where the dark sector is completely decoupled from the SM sector before the DM becomes nonrelativistic and the electroweak phase transition, we should take $g_{*}^{\prime}\left(T_{\mathrm{d}}^{\prime}\right)=2+4(7 / 8)=11 / 2$ 
and $g_{*}\left(T_{\mathrm{d}}^{\prime}\right)=106.75$ and obtain $\Delta N_{\text {eff }}=0.21 \xi^{4}\left(T_{\mathrm{d}}^{\prime}\right)$. Even if we set $\xi\left(T_{\mathrm{d}}^{\prime}\right)=1$, the prediction is consistent with the constraint reported by the Planck data combined with the baryon acoustic oscillation observation and supernova data: $N_{\text {eff }}=3.27 \pm 0.15$ [24]. This marginal preference of $\Delta N_{\text {eff }} \sim 0.2$ originates from the discrepancy in $H_{0}$ between the local and CMB-based measurements. The dark radiation in our model (although not fully) relaxes the $H_{0}$ tension $[25,26]$. We can check the deviation from the SM prediction with a large significance in the near future by, e.g., the CMB-S4 experiment $[27,28]$.

It is also possible that the DM sector is in thermal equilibrium with the SM sector at a high temperature and then decoupled after the DM becomes nonrelativistic. This is the case when the $\mathrm{U}(1)^{\prime}$ gauge boson has a nonzero kinetic mixing with the $\mathrm{U}(1)_{Y}$ gauge boson as we will discuss later. Then we should take $\xi\left(T_{\mathrm{d}}^{\prime}\right)=1$ and $g_{*}^{\prime}\left(T_{\mathrm{d}}^{\prime}\right)=2$. As we will discuss shortly, the decoupling temperature is just below the DM mass, which is of the order of or larger than the electroweak scale. Thus, we expect $g_{*}\left(T_{\mathrm{d}}^{\prime}\right) \simeq 100$, which results in $\Delta N_{\text {eff }} \simeq 0.07$. This scenario is also consistent with the Planck data and would be checked by the CMB-S4 experiment in the future.

\section{DARK MATTER FROM DARK SO $(10)^{\prime}$}

Now we shall provide a UV theory of the DM sector, which is similar to the SM GUT. We introduce an $\mathrm{SO}(10)^{\prime}$ gauge group and a chiral fermion transforming as the 16 representation, assuming that the gauge group is spontaneously broken to $\mathrm{SU}(5)^{\prime} \times \mathrm{U}(1)^{\prime}$ at the energy scale much above $10^{13} \mathrm{GeV}$ and below the Planck scale. After the spontaneous symmetry breaking (SSB), the fermion is decomposed into $\psi, \chi$, and $N$, which transform as the $\overline{\mathbf{5}}$, 10, and 1 representations in the $\mathrm{SU}(5)^{\prime}$ gauge group, respectively. If we denote the $\mathrm{U}(1)^{\prime}$ charge of $N$ as $q$ $(=\sqrt{10} / 4)$, those of $\psi$ and $\chi$ are $-3 q / 5$ and $q / 5$, respectively [29]. If one starts from a generic $\mathrm{SU}(5)^{\prime} \times$ $\mathrm{U}(1)^{\prime}$ gauge theory instead of the $\mathrm{SO}(10)^{\prime}$ gauge theory, the $\mathrm{U}(1)^{\prime}$ charge $q$ may be different from $\sqrt{10} / 4$.

Since the $\mathrm{SU}(5)^{\prime}$ gauge interaction is asymptotically free, it becomes strong and is confined at a dynamical scale $\Lambda_{5}^{\prime}$. Below the confinement scale, there is one massless baryonic state composed of fermions as the 't Hooft anomaly matching condition is satisfied [30,31] (see Refs. [15-17] for other applications of this model). It is a linear combination of $\psi \psi \chi \chi, \psi \chi^{\dagger} \chi^{\dagger}$, and $\chi^{\dagger} \chi^{\dagger} \chi^{\dagger} \chi^{\dagger} \chi^{\dagger}$. Since there is no reason that one particular state dominates, we expect that there is no suppression factor in the coefficients of the linear combination. We note that it can be combined with $N$ to form a Dirac fermion. In fact, we can write down, e.g., the following dimension- 6 operator:

$$
\frac{c}{M_{\mathrm{Pl}}^{2}} \psi \psi \chi \chi N+\text { H.c., }
$$

where $c$ is an $\mathcal{O}(1)$ constant. If one starts from the $\mathrm{SO}(10)^{\prime}$ gauge theory, the exchange of massive $\mathrm{SO}(10)^{\prime}$ gauge bosons induces a dimension- 6 operator like

$$
\frac{-c^{\prime}}{2 M_{\mathrm{GUT}}^{\prime}} \chi^{\dagger} \bar{\sigma}_{\mu} N \chi^{\dagger} \bar{\sigma}^{\mu} \psi+\text { H.c. }
$$

which leads to an operator

$$
\frac{c^{\prime}}{M_{\mathrm{GUT}}^{\prime}{ }^{2}} \chi^{\dagger} \chi^{\dagger} \psi N+\text { H.c., }
$$

through the Fierz transformation. These result in Dirac mass terms below the dynamical scale, and its mass is roughly given by

$$
m_{\eta} \sim c \frac{\left(\Lambda_{5}^{\prime}\right)^{3}}{M_{\mathrm{Pl}}^{2}} \quad \text { or } \quad c^{\prime} \frac{\left(\Lambda_{5}^{\prime}\right)^{3}}{M_{\mathrm{GUT}}^{\prime}},
$$

where we absorbed unknown $\mathcal{O}(1)$ factors into the parameters $c$ and $c^{\prime}$. This is of the order of $100 \mathrm{GeV}-10 \mathrm{TeV}$ when the dynamical scale $\Lambda_{5}^{\prime}$ is of the order of $10^{13-14} \mathrm{GeV}$ and $M_{\mathrm{GUT}}^{\prime}=M_{\mathrm{pl}}$. If $M_{\mathrm{GUT}}^{\prime}$ is of the order of $10^{16} \mathrm{GeV}$, the dynamical scale should be of the order of $10^{11-12} \mathrm{GeV}$. As a result, the low-energy dark sector is nothing but the DM model discussed in the previous section.

One may think that our low-energy dark sector looks different from the one in Ref. [31]. As discussed and shown in the paper, the low-energy spectrum in the Higgs phase is equivalent to the one in the complementary symmetric picture. We use the latter picture, supplemented by the 't Hooft anomaly matching condition. In the former picture, on the other hand, the bilinear fermion condensate $\langle\mathbf{1 0 1 0}\rangle$ breaks $\mathrm{SU}(5)^{\prime}$ to $\mathrm{SU}(4)^{\prime}$ and the $\mathrm{SU}(4)^{\prime}$ theory is confined at a slightly lower energy scale. The $\langle\mathbf{1 0 1 0}\rangle$ condensation breaks the original $\mathrm{U}(1)^{\prime}$, but there remains a $\mathrm{U}(1)^{\prime \prime}$ symmetry with a generator of $T_{24}+T_{\mathrm{U}(1)^{\prime}}$. The remaining $\mathrm{U}(1)^{\prime \prime}$ charge is a linear combination of the original $\mathrm{U}(1)^{\prime}$ charge $\left[-3 / 5\right.$ for $\overline{5}$ of $\mathrm{SU}(5)^{\prime}$ and $1 / 5$ for 10] and a Cartan charge of $\mathrm{SU}(5)^{\prime}$ that is invariant under the $\mathrm{SU}(4)^{\prime}(1 / 10,1 / 10,1 / 10,1 / 10,-2 / 5)$. For example, the $\mathrm{SU}(5)^{\prime}$ multiplet $\overline{\mathbf{5}}$ contains $(\overline{\mathbf{4}},-1 / 2)$ and $(\mathbf{1},-1)$ under $\mathrm{SU}(4)^{\prime} \times \mathrm{U}(1)^{\prime}$, while $\mathbf{1 0}$ contains $(\mathbf{6}, 0)$ and $(\mathbf{4}, 1 / 2)$. The bilinear combination that breaks $\mathrm{SU}(4)^{\prime},\langle\mathbf{4} \overline{\mathbf{4}}\rangle$, is neutral under $\mathrm{U}(1)^{\prime \prime}$ and does not break $\mathrm{U}(1)^{\prime \prime}$. Thus, there remains $\mathrm{U}(1)^{\prime \prime}$ gauge symmetry below the $\mathrm{SU}(4)^{\prime}$ confinement scale. The singlet under $\mathrm{SU}(4)^{\prime}$ has $\mathrm{U}(1)^{\prime \prime}$ charge of -1 and makes a Dirac pair with $N$, which has the opposite $\mathrm{U}(1)^{\prime \prime}$ charge. This is equivalent to the one discussed above, as is consistent with the complementarity of gauge theories. Hereafter, we denote $\mathrm{U}(1)^{\prime \prime}$ as $\mathrm{U}(1)^{\prime}$ for notational simplicity.

As for the SM sector, we consider also an $\mathrm{SO}(10)$ GUT, motivated by the thermal leptogenesis [32] (see, e.g., 


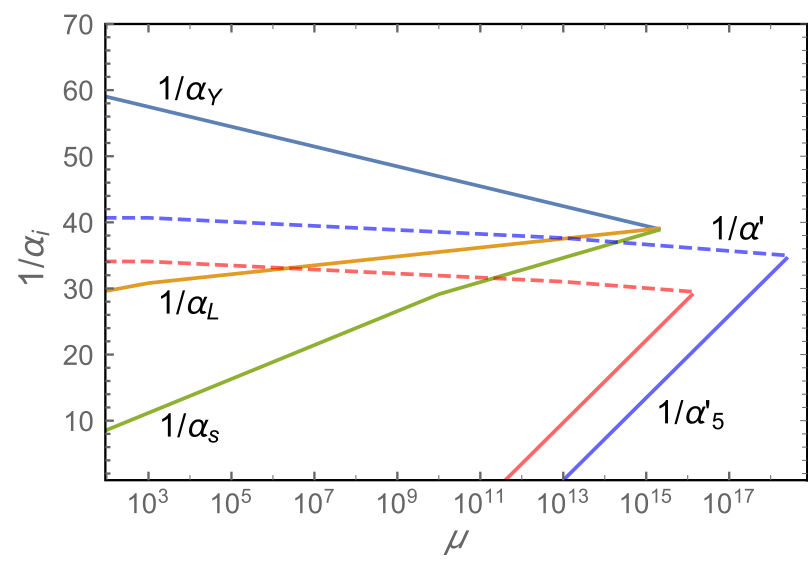

FIG. 2. Renormalization group running of gauge coupling constants, where $\mu$ is the renormalization scale in units of $\mathrm{GeV}$. We introduce adjoint fermions for $\mathrm{SU}(2)_{L}$ and $\mathrm{SU}(3)_{c}$ at $10^{3}$ and $10^{10} \mathrm{GeV}$, respectively, to present an example of gauge coupling unification of the SM gauge interactions [41]. We plot the running of $\alpha^{\prime}$ with $q=\sqrt{10} / 4$ for the case in which it is unified with $\mathrm{SU}(5)^{\prime}$ gauge coupling constant $\alpha_{5}^{\prime}$ at the energy scale of $10^{16} \mathrm{GeV}$ (red dashed line) and the Planck scale (blue dashed line).

Refs. [33-36] for recent reviews) and seesaw mechanism [37-40]. Here, we introduce a right-handed neutrino with mass of the order of or larger than $10^{9} \mathrm{GeV}$ in the $\mathrm{SM}$ sector. $^{2}$ Then, we expect an $\mathrm{SO}(10) \times \mathrm{SO}(10)^{\prime}$ gauge theory to be a unified model of the SM and DM sectors. The similarity of the SM and DM sectors may be because a fermion in the 16 representation is the minimal particle content for the anomaly-free chiral $\mathrm{SO}(10)$ gauge theory.

An example of renormalization group running of gauge coupling constants is shown in Fig. 2, where we note that there are three flavors for quarks and leptons while there is only one "flavor" in the dark sector. Although an explicit construction of the GUT model in the SM sector is beyond the scope of this paper, we present a gauge coupling unification in a simple GUT model proposed in Ref. [41]. They introduced adjoint fermions for $\mathrm{SU}(3)_{c}$ and $\mathrm{SU}(2)_{L}$ at an intermediate scale and at the $\mathrm{TeV}$ scale, respectively. ${ }^{3}$ Although the $\mathrm{SU}(2)_{L}$ adjoint fermion is stable, we assume that it is a subdominant component of DM or there is another field that makes it unstable. Noting that this is just one example of GUT in the SM sector, we plot the gauge coupling unification in the simplest case in the figure. We do not

\footnotetext{
${ }^{2}$ We introduce a Higgs field with a 16 representation, which contains a U(1) ${ }_{\mathrm{B}-\mathrm{L}}$ charged Higgs after the $\mathrm{SSB}$ of $\mathrm{SO}(10)$. We assume that it spontaneously breaks the $\mathrm{U}(1)_{\mathrm{B}-\mathrm{L}}$ gauge symmetry to give the mass for the right-handed neutrino.

${ }^{3}$ The adjoint fermions may come from a fermion in the 45 representation under $\mathrm{SO}(10)$. The mass terms for the adjoint fermions do not break the gauge symmetries, so that we can add them without introducing light Higgs fields, which potentially change the renormalization group running for the gauge coupling constants.
}

introduce such adjoint fermions in the dark sector, or we assume that they are heavier than the dynamical scale if present.

We are interested in the case where $q=\sqrt{10} / 4$ and the $\mathrm{SU}(5)^{\prime}$ gauge coupling $\alpha_{5}^{\prime}$ becomes strong at $\Lambda_{5}^{\prime} \sim$ $10^{11-13} \mathrm{GeV}$. Starting from $\alpha^{\prime} \simeq 2.9 \times 10^{-2}\left(2.5 \times 10^{-2}\right)$ at the electroweak scale and assuming $\Lambda_{5}^{\prime}=4 \times 10^{11} \mathrm{GeV}$ $\left(10^{13} \mathrm{GeV}\right)$, we find that the $\mathrm{SU}(5)^{\prime} \times \mathrm{U}(1)^{\prime}$ gauge group can be unified at the energy scale of $M_{\mathrm{GUT}}^{\prime}=10^{16} \mathrm{GeV}$ $\left(2.4 \times 10^{18} \mathrm{GeV}\right)$. The running gauge coupling constants for $\mathrm{SU}(5)^{\prime}$ and $\mathrm{U}(1)^{\prime}$ are shown as the red (blue) solid and dashed lines in Fig. 2, respectively. The gauge coupling constant at the electroweak scale is plotted as the upper (lower) dashed line in Fig. 1. It shows that the DM mass should be about $700 \mathrm{GeV}(600 \mathrm{GeV})$ to explain the observed amount of DM if $\xi\left(T_{\mathrm{d}}^{\prime}\right)=1$.

We note that the gauge coupling constants in the dark sector do not need to be unified at the same scale as the GUT scale in the SM but can be unified at the energy scale between the dynamical scale $\Lambda_{5}^{\prime}$ and the Planck scale. However, we expect that the gauge coupling constant at the unification scale is of the same order as that of the SM gauge coupling constants and, hence, $M_{\mathrm{GUT}}^{\prime}=\mathcal{O}\left(10^{16-18}\right) \mathrm{GeV}$. In this case, $\alpha^{\prime}$ must be within the region between the dashed lines in Fig. 1, namely,

$$
\alpha^{\prime}=(2.5-2.9) \times 10^{-2}, \quad m_{\eta}=0.6-0.7 \mathrm{TeV} .
$$

This is the prediction of the chiral $\mathrm{SO}(10)^{\prime}$ gauge theory in the DM sector.

\section{KINETIC MIXING}

Finally, we comment on the kinetic mixing between the $\mathrm{U}(1)_{Y}$ and $\mathrm{U}(1)^{\prime}$ gauge bosons. For this purpose, we need to specify how to break the gauge groups at the GUT scale. We first note that a scalar field transforming as the 45 representation in $\mathrm{SO}(10)$ is decomposed into scalar fields in the $\mathbf{1}+\mathbf{1 0}+\overline{\mathbf{1 0}}+\mathbf{2 4}$ representations under an $\mathrm{SU}(5)$ [C SO(10)] gauge group. The singlet 1 can be used to break $\mathrm{SO}(10)$ to $\mathrm{SU}(5) \times \mathrm{U}(1)$. We assume that $\mathrm{SO}(10)$ and $\mathrm{SO}(10)^{\prime}$ are spontaneously broken to $\mathrm{SU}(5) \times \mathrm{U}(1)_{(B-L)}$ and $\mathrm{SU}(5)^{\prime} \times \mathrm{U}(1)^{\prime}$ by nonzero vacuum expectation values (VEVs) of $\mathbf{4 5}_{H}$ and $\mathbf{4 5}_{H}^{\prime}$, respectively. The remaining $\mathrm{SU}(5)$ in the SM sector is also assumed to be spontaneously broken to the $\mathrm{SM}$ gauge group $\mathrm{G}_{\mathrm{SM}}$ by the field in the 24 representation that is contained in $\mathbf{4 5}_{H}$. On the other hand, we assume that $\mathbf{2 4}^{\prime}$ in $\mathbf{4 5}_{H}^{\prime}$ has a vanishing VEV. We finally obtain $\mathrm{G}_{\mathrm{SM}} \times \mathrm{U}(1)_{(B-L)} \times \mathrm{SU}(5)^{\prime} \times U(1)^{\prime}$ below these energy scales. The $\mathrm{U}(1)_{(B-L)}$ is assumed to be spontaneously broken at an intermediate scale to give a nonzero mass to the right-handed neutrinos.

Then, even if we start from the $\mathrm{SO}(10) \times \mathrm{SO}(10)^{\prime}$ gauge theory, the kinetic mixing between $\mathrm{U}(1)_{Y}$ and $\mathrm{U}(1)^{\prime}$ is induced from the following dimension- 6 operator: 


$$
\frac{c^{\prime \prime}}{M_{\mathrm{Pl}}^{2}} \mathbf{4 5}_{H}\left(F_{10}\right)_{\mu \nu} \mathbf{4 5}_{H}^{\prime}\left(F_{10^{\prime}}^{\prime}\right)^{\mu \nu}
$$

where $c^{\prime \prime}$ is an $\mathcal{O}(1)$ constant and $F_{10}$ and $F_{10^{\prime}}^{\prime}$ are the field strengths of $\mathrm{SO}(10)$ and $\mathrm{SO}(10)^{\prime}$, respectively. The kinetic mixing parameter is of the order of $\epsilon \sim c^{\prime \prime}\left(v_{\mathrm{GUT}} / M_{\mathrm{Pl}}\right)$ $\left(v^{\prime} / M_{\mathrm{Pl}}\right)$, where $v_{\mathrm{GUT}}$ and $v^{\prime}$ are the VEVs of $\mathbf{2 4}\left(\subset \mathbf{4 5}_{H}\right)$ and $\mathbf{4 5}_{H}^{\prime}$, respectively. We expect that the dark $\mathrm{SO}(10)^{\prime}$ is spontaneously broken between the energy scale of $10^{16} \mathrm{GeV}$ and the Planck scale. Therefore, the factor of $v^{\prime} / M_{\mathrm{Pl}}$ can be $\mathcal{O}\left(10^{-2}-1\right)$, and, hence, $\epsilon$ is $\mathcal{O}\left(10^{-(3-6)}\right)$ for $c^{\prime \prime}=0.1-1$.

The dark photon $\gamma^{\prime}$ can be in thermal equilibrium with the SM sector by the annihilation and inverse-annihilation processes of DM into the SM particles $f \bar{f} \leftrightarrow \eta \bar{\eta}$, the Compton scattering process $\eta \gamma \leftrightarrow \eta \gamma\left(\gamma^{\prime}\right)$, and the Coulomb scattering process $f \eta \leftrightarrow f \eta$ via the kinetic mixing, where $f$ represents generic SM particles with nonzero $\mathrm{U}(1)_{Y}$ charges. Comparing the energy transfer rate $\Gamma$ with the Hubble expansion rate $H$, we find that these processes are most important at the temperature around the DM mass. The ratio at $T \sim m_{\eta}$ is roughly given by

$$
\frac{\Gamma}{H} \sim \frac{\epsilon^{2} q^{2} \alpha \alpha^{\prime} n_{f}}{m_{\eta}^{2} H\left(m_{\eta}\right)} \sim\left(\frac{\epsilon}{10^{-6}}\right)^{2}\left(\frac{q^{2} \alpha^{\prime}}{0.02}\right)\left(\frac{m_{\eta}}{1 \mathrm{TeV}}\right)^{-1},
$$

where $n_{f}$ is the number density of the SM particles with nonzero $\mathrm{U}(1)_{Y}$ charges. The ratio is larger than of the order of unity when $\epsilon \gtrsim 10^{-6}$ for $m_{\eta}=1 \mathrm{TeV}$. This process freezes out soon after the DM becomes nonrelativistic, that is, around the temperature of $\mathcal{O}(0.1) m_{\eta}$. Therefore, if the kinetic mixing is not strongly suppressed, the temperature of the DM sector is the same as the SM sector around the time of DM freezeout, and we should take $\xi\left(T_{\mathrm{d}}^{\prime}\right)=1$.

The nonzero kinetic mixing between the $\mathrm{U}(1)_{Y}$ [or $\left.\mathrm{U}(1)_{\mathrm{EM}}\right]$ and $\mathrm{U}(1)^{\prime}$ gauge bosons leads to a rich phenomenology for the DM detection experiments. It is convenient to diagonalize the gauge bosons in the basis that the $\mathrm{SM}$ particles are charged only under $\mathrm{U}(1)_{\mathrm{EM}}$ and the $\mathrm{DM}$ is charged under both $\mathrm{U}(1)_{\mathrm{EM}}$ and $\mathrm{U}(1)^{\prime}$. The effective electromagnetic charge of DM is given by $q_{\mathrm{eff}}=$ $-\epsilon q e^{\prime} \cos \theta_{W} / e_{\mathrm{EM}}$, where $e_{\mathrm{EM}}$ is the gauge coupling of $\mathrm{U}(1)_{\mathrm{EM}}$ and $\theta_{W}$ is the Weinberg angle. The direct detection experiments for DM put a stringent constraint on such a millicharged DM [42,43]. However, the constraint is not applicable to the DM with a relatively large charge, because the DM loses its kinetic energy in the atmosphere [44]. The measurement of CMB temperature anisotropies also constrains the millicharged DM for a larger charge region $[45,46]$. In combination, there is an allowed range such as ${ }^{4}$

\footnotetext{
${ }^{4} \mathrm{~A}$ much stronger constraint may be derived by requiring that the millicharged DM does not diffuse within galactic clusters [47], though simulations may be required to correctly take into account the nonlinear gravity effect $[48,49]$.
}

$10^{-6}\left(\frac{m_{\eta}}{10^{3} \mathrm{GeV}}\right) \lesssim \epsilon \lesssim 3 \times 10^{-5}\left(\frac{m_{\eta}}{10^{3} \mathrm{GeV}}\right)^{1 / 2}$.

This can be consistent with the $\mathrm{SO}(10) \times \mathrm{SO}(10)^{\prime}$ model, because $\epsilon=\mathcal{O}\left(10^{-(3-6)}\right)$ depending on the SSB scale of $\mathrm{SO}(10)^{\prime}$.

Finally, we comment on the case in which the kinetic mixing is as small as $10^{-(10-11)}$. Such a small kinetic mixing can be realized if there is Pati-Salam symmetry for the SM sector at an intermediate scale and the VEV of $24\left(\subset \mathbf{4 5}_{H}\right)$ is much smaller than the GUT scale, or $c^{\prime \prime} \simeq 10^{-6}$. In this case, the DM sector is completely decoupled from the SM sector even in the early Universe, and the ratio of the temperatures in these sectors is determined solely by the branching ratio of the inflaton decay into these sectors. We note that the gauge-couplingmass relation of DM, which is shown as the blue curve in Fig. 1, changes only of the order of $\sqrt{\xi\left(T_{d}^{\prime}\right)}$ unless the Sommerfeld enhancement effect is strongly efficient. The constraint by the direct detection experiment of DM for such a very small kinetic mixing is given by $\epsilon \lesssim$ $10^{-10}\left(m_{\eta} / 1 \mathrm{TeV}\right)^{1 / 2}$ for $m_{\eta} \gtrsim 100 \mathrm{GeV}[43,50]$. This constraint will be improved by the LUX-ZEPLIN experiment for 1000 days by a factor of about 10 [51].

\section{DISCUSSION}

We have proposed a chiral $\mathrm{SO}(10)^{\prime}$ gauge theory as a UV theory of light Dirac DM that is charged under the dark $\mathrm{U}(1)^{\prime}$ gauge symmetry. The darkly charged $\mathrm{DM}$ is also considered as double-disk DM, though it must be a subdominant component [52-54]. A similar model with a nonzero kinetic mixing between $\mathrm{U}(1)^{\prime}$ and the electroweak U(1) gauge bosons, namely, the millicharged (or minicharged) DM model, is also motivated by the absorption profile around $78 \mathrm{MHz}$ in the sky-averaged spectrum of $21 \mathrm{~cm}$ line by the Experiment to Detect the Global EoR Signature (EDGES) experiment [55-61]. The DM with a massive $\mathrm{U}(1)^{\prime}$ gauge boson is also considered in Refs. [17,21,62-71]. Our $\mathrm{SO}(10)^{\prime}$ gauge theory may also be a natural candidate for the UV theory of those models.

DM has a self-interaction mediated by the gauge boson. The cross section is velocity dependent, which is supported by the observations of DM halos in galaxy and galaxy cluster scales. As DM couples to the SM sector only via the small kinetic mixing, the gravitational search is one of the important DM searches in our model (see, e.g., Ref. [72]). It would be interesting to collect a larger number of samples in different length scales so that we can determine the velocity dependence on the selfinteraction cross section [21,73]. This may allow us to distinguish our model from the self-interacting DM model with a velocity-independent cross section, like the ones studied in Refs. [74-79]. It is also worth investigating if 
the self-interacting DM with a massless vector mediator solves the small-scale issues for cosmological structure formation [23,80-82].

\section{ACKNOWLEDGMENTS}

We thank an anonymous referee for pointing out the existence of the GUT' suppressed dimension- 6 operator. A. K. was supported by Institute for Basic Science under Project Code No. IBS-R018-D1. A. K. acknowledges the Mainz Institute for Theoretical Physics (MITP) of the Cluster of Excellence PRISMA+ (Project ID No. 39083149) for enabling A. K. to complete a significant portion of this work. T. T. Y. was supported in part by the China Grant for Talent Scientific Start-Up Project and the JSPS Grant-in-Aid for Scientific Research No. 16H02176, No. 17H02878, and No. 19H05810 and by World Premier International Research Center Initiative (WPI Initiative), MEXT, Japan. T. T. Y. thanks Hamamatsu Photonics.
[1] J. R. Ellis, S. Kelley, and D. V. Nanopoulos, Phys. Lett. B 249, 441 (1990).

[2] J. R. Ellis, S. Kelley, and D. V. Nanopoulos, Phys. Lett. B 260, 131 (1991).

[3] U. Amaldi, W. de Boer, and H. Furstenau, Phys. Lett. B 260, 447 (1991).

[4] C. Giunti, C. W. Kim, and U. W. Lee, Mod. Phys. Lett. A 06, 1745 (1991).

[5] P. Langacker and M.-X. Luo, Phys. Rev. D 44, 817 (1991).

[6] D. Chang, R. N. Mohapatra, J. Gipson, R. E. Marshak, and M. K. Parida, Phys. Rev. D 31, 1718 (1985).

[7] N. G. Deshpande, E. Keith, and P. B. Pal, Phys. Rev. D 46, 2261 (1992).

[8] M. Fukugita and T. Yanagida, Physics of Neutrinos and Applications to Astrophysics (Springer-Verlag, Berlin, 2003).

[9] S. Bertolini, L. Di Luzio, and M. Malinský, Phys. Rev. D 81, 035015 (2010).

[10] Y. Mambrini, K. A. Olive, J. Quevillon, and B. Zaldívar, Phys. Rev. Lett. 110, 241306 (2013).

[11] Y. Mambrini, N. Nagata, K. A. Olive, J. Quevillon, and J. Zheng, Phys. Rev. D 91, 095010 (2015).

[12] J. L. Feng, M. Kaplinghat, H. Tu, and H.-B. Yu, J. Cosmol. Astropart. Phys. 07 (2009) 004.

[13] P. Agrawal, F.-Y. Cyr-Racine, L. Randall, and J. Scholtz, J. Cosmol. Astropart. Phys. 05 (2017) 022.

[14] R. Balkin, M. Ruhdorfer, E. Salvioni, and A. Weiler, J. Cosmol. Astropart. Phys. 11 (2018) 050.

[15] N. Arkani-Hamed and Y. Grossman, Phys. Lett. B 459, 179 (1999).

[16] M. B. Gavela, M. Ibe, P. Quilez, and T. T. Yanagida, Eur. Phys. J. C 79, 542 (2019).

[17] A. Kamada, M. Yamada, and T. T. Yanagida, J. High Energy Phys. 07 (2019) 180.

[18] P. Gondolo and G. Gelmini, Nucl. Phys. B360, 145 (1991).

[19] B. von Harling and K. Petraki, J. Cosmol. Astropart. Phys. 12 (2014) 033.

[20] D. A. Buote, T. E. Jeltema, C. R. Canizares, and G. P. Garmire, Astrophys. J. 577, 183 (2002).

[21] M. Kaplinghat, S. Tulin, and H.-B. Yu, Phys. Rev. Lett. 116, 041302 (2016).

[22] K.-J. Ahn and P. R. Shapiro, J. Korean Astron. Soc. 36, 89 (2003).
[23] K.-J. Ahn and P. R. Shapiro, Mon. Not. R. Astron. Soc. 363, 1092 (2005).

[24] N. Aghanim et al. (Planck Collaboration), arXiv:1807 .06209

[25] J. L. Bernal, L. Verde, and A. G. Riess, J. Cosmol. Astropart. Phys. 10 (2016) 019.

[26] N. Arendse et al., Astron. Astrophys. 639, A57 (2020).

[27] W. L. K. Wu, J. Errard, C. Dvorkin, C. L. Kuo, A. T. Lee, P. McDonald, A. Slosar, and O. Zahn, Astrophys. J. 788, 138 (2014).

[28] K. N. Abazajian et al. (CMB-S4 Collaboration), arXiv:1610 .02743

[29] P. Pachołek, Eur. Phys. J. C 73, 2436 (2013).

[30] G. 't Hooft, NATO Sci. Ser. B 59, 135 (1980).

[31] S. Dimopoulos, S. Raby, and L. Susskind, Nucl. Phys. B173, 208 (1980).

[32] M. Fukugita and T. Yanagida, Phys. Lett. B 174, 45 (1986).

[33] W. Buchmüller, P. Di Bari, and M. Plümacher, Nucl. Phys. B643, 367 (2002); 793, 362(E) (2008).

[34] G. F. Giudice, A. Notari, M. Raidal, A. Riotto, and A. Strumia, Nucl. Phys. B685, 89 (2004).

[35] W. Buchmüller, R. D. Peccei, and T. Yanagida, Annu. Rev. Nucl. Part. Sci. 55, 311 (2005).

[36] S. Davidson, E. Nardi, and Y. Nir, Phys. Rep. 466, 105 (2008).

[37] P. Minkowski, Phys. Lett. 67B, 421 (1977).

[38] T. Yanagida, Conf. Proc. C 7902131, 95 (1979).

[39] S. L. Glashow, NATO Sci. Ser. B 61, 687 (1980).

[40] M. Gell-Mann, P. Ramond, and R. Slansky, Conf. Proc. C 790927, 315 (1979).

[41] T. Aizawa, M. Ibe, and K. Kaneta, Phys. Rev. D 91, 075012 (2015).

[42] D. S. Akerib et al. (LUX Collaboration), Phys. Rev. Lett. 112, 091303 (2014).

[43] E. Aprile et al. (XENON Collaboration), Phys. Rev. Lett. 121, 111302 (2018).

[44] S. Dimopoulos, D. Eichler, R. Esmailzadeh, and G. D. Starkman, Phys. Rev. D 41, 2388 (1990).

[45] A. Kamada, K. Kohri, T. Takahashi, and N. Yoshida, Phys. Rev. D 95, 023502 (2017).

[46] W. L. Xu, C. Dvorkin, and A. Chael, Phys. Rev. D 97, 103530 (2018).

[47] K. Kadota, T. Sekiguchi, and H. Tashiro, arXiv:1602.04009. 
[48] C. Spethmann, H. Veermäe, T. Sepp, M. Heikinheimo, B. Deshev, A. Hektor, and M. Raidal, Astron. Astrophys. 608, A125 (2017).

[49] S. I. Alvis et al. (Majorana Collaboration), Phys. Rev. Lett. 120, 211804 (2018).

[50] T. Hambye, M. H. G. Tytgat, J. Vandecasteele, and L. Vanderheyden, Phys. Rev. D 98, 075017 (2018).

[51] D. S. Akerib et al. (LUX-ZEPLIN Collaboration), Phys. Rev. D 101, 052002 (2020).

[52] J. Fan, A. Katz, L. Randall, and M. Reece, Phys. Dark Universe 2, 139 (2013).

[53] J. Fan, A. Katz, L. Randall, and M. Reece, Phys. Rev. Lett. 110, 211302 (2013).

[54] M. McCullough and L. Randall, J. Cosmol. Astropart. Phys. 10 (2013) 058.

[55] J. D. Bowman, A. E. E. Rogers, R. A. Monsalve, T. J. Mozdzen, and N. Mahesh, Nature (London) 555, 67 (2018).

[56] J. B. Muñoz and A. Loeb, Nature (London) 557, 684 (2018).

[57] A. Berlin, D. Hooper, G. Krnjaic, and S. D. McDermott, Phys. Rev. Lett. 121, 011102 (2018).

[58] R. Barkana, N. J. Outmezguine, D. Redigolo, and T. Volansky, Phys. Rev. D 98, 103005 (2018).

[59] T. R. Slatyer and C.-L. Wu, Phys. Rev. D 98, 023013 (2018).

[60] H. Liu and T. R. Slatyer, Phys. Rev. D 98, 023501 (2018).

[61] E. D. Kovetz, V. Poulin, V. Gluscevic, K. K. Boddy, R. Barkana, and M. Kamionkowski, Phys. Rev. D 98, 103529 (2018).

[62] S. Tulin, H.-B. Yu, and K. M. Zurek, Phys. Rev. Lett. 110, 111301 (2013).

[63] B. Dasgupta and J. Kopp, Phys. Rev. Lett. 112, 031803 (2014).

[64] T. Bringmann, J. Hasenkamp, and J. Kersten, J. Cosmol. Astropart. Phys. 07 (2014) 042.
[65] P. Ko and Y. Tang, Phys. Lett. B 739, 62 (2014).

[66] J. F. Cherry, A. Friedland, and I. M. Shoemaker, arXiv:141 1.1071 .

[67] T. Kitahara and Y. Yamamoto, Phys. Rev. D 95, 015008 (2017).

[68] E. Ma, Phys. Lett. B 772, 442 (2017).

[69] A. Kamada, K. Kaneta, K. Yanagi, and H.-B. Yu, J. High Energy Phys. 06 (2018) 117.

[70] O. Balducci, S. Hofmann, and A. Kassiteridis, arXiv:1810 .07198

[71] A. Kamada, M. Yamada, and T. T. Yanagida, J. High Energy Phys. 03 (2019) 021.

[72] M. R. Buckley and A.H. G. Peter, Phys. Rep. 761, 1 (2018).

[73] S. Tulin and H.-B. Yu, Phys. Rep. 730, 1 (2018).

[74] Y. Hochberg, E. Kuflik, T. Volansky, and J. G. Wacker, Phys. Rev. Lett. 113, 171301 (2014).

[75] Y. Hochberg, E. Kuflik, H. Murayama, T. Volansky, and J. G. Wacker, Phys. Rev. Lett. 115, 021301 (2015).

[76] H. M. Lee and M.-S. Seo, Phys. Lett. B 748, 316 (2015).

[77] Y. Hochberg, E. Kuflik, and H. Murayama, J. High Energy Phys. 05 (2016) 090.

[78] A. Kamada, M. Yamada, T. T. Yanagida, and K. Yonekura, Phys. Rev. D 94, 055035 (2016).

[79] S.-M. Choi, Y. Hochberg, E. Kuflik, H. M. Lee, Y. Mambrini, H. Murayama, and M. Pierre, J. High Energy Phys. 10 (2017) 162.

[80] S. Balberg, S. L. Shapiro, and S. Inagaki, Astrophys. J. 568, 475 (2002).

[81] J. Koda and P. R. Shapiro, Mon. Not. R. Astron. Soc. 415, 1125 (2011).

[82] R. Essig, S. D. Mcdermott, H.-B. Yu, and Y.-M. Zhong, Phys. Rev. Lett. 123, 121102 (2019). 